\title{
Production Performance Appraisal Rating for Reservoir Management Units Based on Fuzzy Clustering
}

\author{
An-Qi Li, ${ }^{1}$ Yan-Rong Chang, ${ }^{1}$ and Xin-Hai Kong ${ }^{2}$ \\ ${ }^{1}$ Changqing Oilfield Branch, PetroChina Company Limited, Shanxi, Xi'an 710018, China \\ ${ }^{2}$ Department of Graduate, Southwest Petroleum University, Sichuan, Chengdu 610500, China
}

Correspondence should be addressed to Xin-Hai Kong, kongxinhai2@163.com

Received 13 July 2012; Accepted 2 September 2012

Academic Editor: Salvatore Sessa

Copyright (C) 2012 An-Qi Li et al. This is an open access article distributed under the Creative Commons Attribution License, which permits unrestricted use, distribution, and reproduction in any medium, provided the original work is properly cited.

\begin{abstract}
In view of the existing situation of oilfield development, one kind of method to evaluate the production performance of reservoir management units (RMUs) was presented in this paper. Among the commonly used indicators of oilfield development, select 12 indicators from the three aspects of production task, production technology, and reservoir development. According to the principle of fuzzy analytic hierarchy process (FAHP), this paper introduced one kind of new method to get the weights of indicators. By means of the method of TOPSIS, it is easy to obtain the rankings for all the RMUs through calculating the weighted Euclidean distance between each RMU and the positive or negative ideal RMU. Considering the gap between the differences in RMUs, the production performance appraisal ratings of RMUs are determined by fuzzy clustering. This evaluation method could constantly improve the management level of reservoir units and deepen the delicacy management of oilfield development.
\end{abstract}

\section{Introduction}

The oilfield companies mostly take the management concept of "Benchmarking" during the process of oilfield development [1]. According to the dynamic analysis of reservoir development, the technical section provides a kind of development scheme and sets some feasible goals that should be achieved. And the reservoir management units (RMUs) must achieve the production goals in accordance with the development requirements [2]. Currently, the development department in the process of oilfield development evaluates the production performance of the reservoir management units (RMUs) based on their own statistics data and the assessment results calculated by themselves [3]. It means that the evaluation accuracy is not high enough and the crosswise contrast is not enough. In order to make the development department accurately and timely, grasp the current situation of development and management of RMUs, it needs to establish a relatively perfect evaluation system to really respond to the management level, efficiency, and development effect of RMUs, promoting the delicacy management of oilfield development. In this paper, we first present the evaluation indicators and their computing method. In order to reasonably decide the weight of each indicator, a kind of fuzzy AHP is introduced in Section 3. Next, we introduce the method of TOPSIS to decide the comprehensive ranking of RMUs and use the Euclidean distance to describe the proximity between two RMUs. However, the proximity among the RMUs is different, so we adopt the fuzzy clustering to classify the RMUs. In order to obtain the optimal classification, the $F$-statistics is mentioned in Section 4.

\section{Evaluation Indicators and Their Computing Method}

Through the analysis, the production performance evaluation indicators of RMU are divided into three aspects of production task, reservoir development, and production technology [1-5] (see Figure 1), including 12 indicators in the following.

2.1. Production Task. The production task $[4,5]$, denoted as $u_{1}$, contains the task completion rate of crude oil $\left(u_{11}\right)$ and the task completion rate of water injection $\left(u_{12}\right)$. 


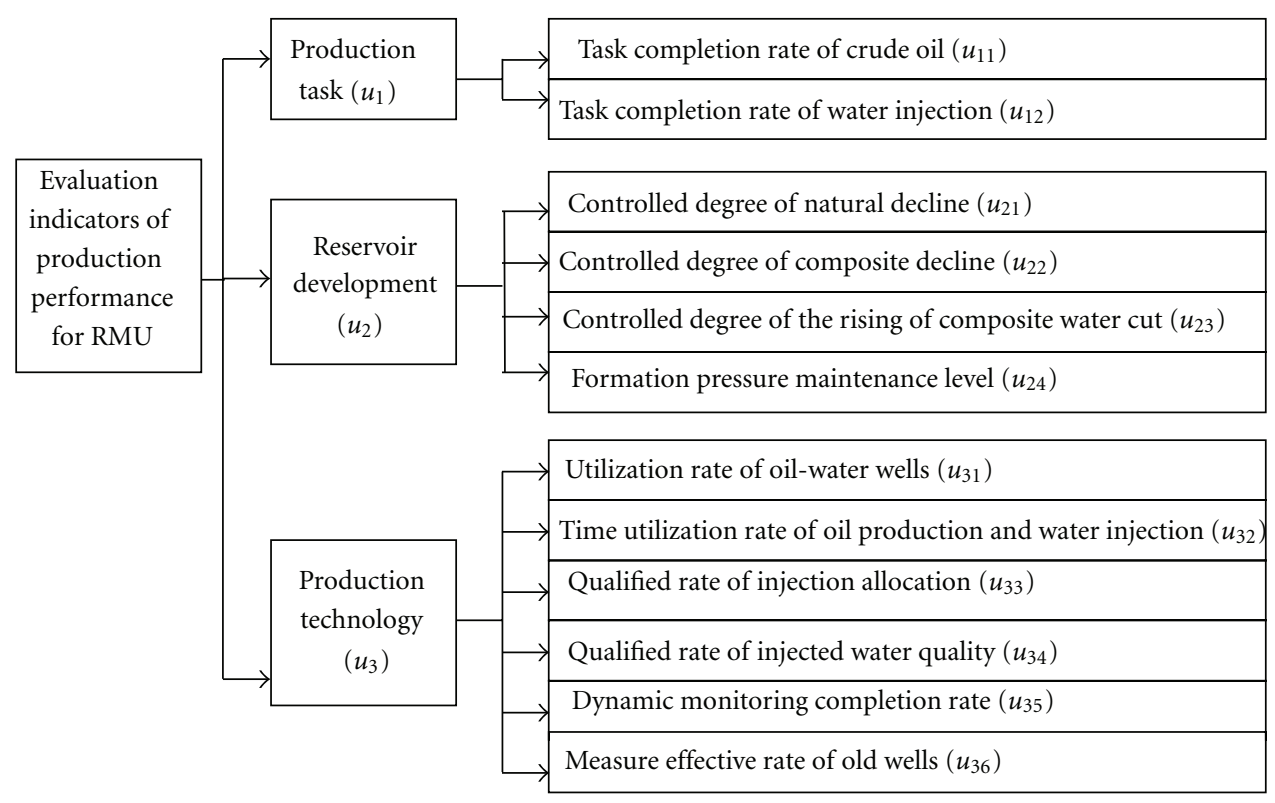

Figure 1: Hierarchical relationship of the evaluation indicators.

(1) Task Completion Rate of Crude Oil. $u_{11}=q_{o} / \tilde{q}_{o} \times 100 \%$, where $q_{o}$ is the actual production of crude oil and $\tilde{q}_{o}$ is the planned output of crude oil, unit: "tons".

(2) Task Completion Rate of Water Injection. $u_{12}=q_{w} / \widetilde{q}_{w} \times$ $100 \%$, where $q_{w}$ is the actual volume of water injection and $\tilde{q}_{w}$ is the geology-required volume of water injection, unit: $\mathrm{m}^{3}$.

2.2. Reservoir Development. The Reservoir development [6, $7]$, denoted as $u_{2}$, contains the controlled degree of natural decline $\left(u_{21}\right)$, the controlled degree of composite decline $\left(u_{22}\right)$, the controlled degree of the rising of composite water cut $\left(u_{23}\right)$, and the formation pressure maintenance level $\left(u_{24}\right)$.

(1) Controlled Degree of Natural Decline. $u_{21}=\left(\widetilde{D}_{r}-\right.$ $\left.D_{r}\right) / \tilde{D}_{r} \times 100 \%$, where $D_{r}$ is the actual natural decline rate and $\widetilde{D}_{r}$ is the control target of natural decline rate, unit: $\%$.

(2) Controlled Degree of Composite Decline. $u_{22}=\left(\widetilde{D}_{t}-\right.$ $\left.D_{t}\right) / \widetilde{D}_{t} \times 100 \%$, where $D_{t}$ is the actual composite decline rate and $\widetilde{D}_{t}$ is the control target of composite decline rate, unit: $\%$.

(3) Controlled Degree of the Rising of Composite Water Cut. $u_{23}=\left(\Delta \tilde{f}_{w}-\Delta f_{w}\right) / \Delta \tilde{f}_{w} \times 100 \%$, where $\Delta f_{w}$ is the actual rising rate of composite water cut and $\Delta \tilde{f}_{w}$ is the control target of the raising rate of composite water cut, unit: $\%$.

(4) Formation Pressure Maintenance Level. $u_{24}=p / p_{0} \times$ $100 \%$, where $p$ is the current formation pressure and $p_{0}$ is the original formation pressure, unit: "MPa".
2.3. Production Technology. The production technology [8], denoted as $u_{3}$, contains the utilization rate of oil-water wells $\left(u_{31}\right)$, the hour utilization rate of oil production, and water injection $\left(u_{32}\right)$, the qualified rate of injection allocation $\left(u_{33}\right)$, the qualified rate of injected water quality $\left(u_{34}\right)$, the dynamic monitoring completion rate $\left(u_{35}\right)$, and the measure effective rate of old wells $\left(u_{36}\right)$.

(1) Utilization Rate of Oil-Water Wells. $u_{31}^{o}=n_{o} / \tilde{n}_{o} \times 100 \%$, where $u_{31}^{o}$ is the utilization rate of oil wells, $n_{o}$ is the active number of oil wells, and $\tilde{n}_{o}$ is the total number of oil wells; and $u_{31}^{w}=n_{w} / \tilde{n}_{w} \times 100 \%$, where $u_{31}^{w}$ is the utilization rate of water injection wells, $n_{w}$ is the active number of water injection wells, and $\tilde{n}_{w}$ is the total number of water injection wells. Therefore, the utilization rate of oil-water wells can be defined by

$$
u_{31}=\frac{\tilde{n}_{o}}{\tilde{n}_{o}+\tilde{n}_{w}} \times u_{31}^{o}+\frac{\tilde{n}_{w}}{\tilde{n}_{o}+\tilde{n}_{w}} \times u_{31}^{w} .
$$

(2) Time Utilization Rate of Oil Production and Water Injection. $u_{32}^{o}=T_{o} / \widetilde{T}_{o} \times 100 \%$, where $u_{32}^{o}$ is the time utilization rate of oil production, $T_{o}$ is the actual time of oil production and $\widetilde{T}_{o}$ is the calendar time of oil production; $u_{32}^{w}=T_{w} / \tilde{T}_{w} \times 100 \%$, where $u_{32}^{w}$ is the time utilization rate of water injection, $T_{w}$ is the actual time of water injection, and $\widetilde{T}_{w}$ is the calendar time of water injection, unit: "day". Therefore, the time utilization rate of oil production and water injection can be defined by

$$
u_{32}=\frac{n_{o}}{n_{o}+n_{w}} \times u_{32}^{o}+\frac{n_{w}}{n_{o}+n_{w}} \times u_{32}^{w}
$$

(3) Qualified Rate of Injection Allocation. $u_{33}=c_{w} / \tilde{c}_{w} \times$ $100 \%$, where $\widetilde{c}_{w}$ is the sum of the number of wells and the 
number of layers for water injection allocation and $c_{w}$ is the sum of the qualified wells and the qualified layers.

(4) Qualified Rate of Injected Water Quality. $u_{34}=m_{w} / \tilde{m}_{w} \times$ $100 \%$, where $\tilde{m}_{w}$ is the total number of water quality monitoring and $m_{w}$ is the number of qualified water sample.

(5) Dynamic Monitoring Completion Rate. $u_{35}^{j}=k_{j} / \tilde{k}_{j} \times$ $100 \%$ is the dynamic monitoring completion rate of the $j$ th project, and the comprehensive dynamic monitoring completion rate is defined as

$$
u_{35}=\sum_{j=1}^{n}\left(\frac{\tilde{k}_{j}}{\tilde{K}} \times u_{35}^{j}\right),
$$

where $n$ is the number of monitoring project, $\widetilde{K}$ is the total number of planned monitoring, $\tilde{k}_{j}$ is the number of planned monitoring for the $j$ th project, and $k_{j}$ is the actual number of monitoring for the $j$ th project, unit: times.

(6) Measure Effective Rate of Old Wells. $u_{36}=n_{\mathrm{eff}} / \tilde{n} \times 100 \%$, where $\tilde{n}$ is the total number of measures and $n_{\text {eff }}$ is the number of effective measures.

\section{The Method to Determine the Weights of Evaluation Indicators}

At present, with regard to determining the weights of evaluation indicators, the analytic hierarchy process (AHP) is a kind of relatively ideal method. While the traditional AHP needs to do the consistency test and constantly adjust the judgment matrix, some scholars put forward the fuzzy analytic hierarchy process (FAHP) [9-18]. We introduce a kind of fuzzy AHP to determine the weights of indicators in this section. The principle is as follows.

Definition 1 (see [9-11]). Assume that $A$ is an $n$-order matrix, denoted as $A=\left(a_{i j}\right)_{n \times n}$,

(a) $A$ is called a fuzzy matrix if for all $i, j \in\{1,2, \ldots, n\}$, $A$ satisfies $0 \leq a_{i j} \leq 1$

(b) $A$ is called a fuzzy complementary matrix if for all $i, j \in\{1,2, \ldots, n\}, A$ also satisfies $a_{i j}+a_{j i}=1$;

(c) $A$ is called a fuzzy consistent judgment matrix if for any $i, j, k \in\{1,2, \ldots, n\}, A$ further satisfies $a_{i j}=a_{i k}-$ $a_{j k}+0.5$.

Property 1. $A$ is a fuzzy consistent judgment matrix if and only if for any $i, j \in\{1,2, \ldots, n\}$ and all $k \in\{1,2, \ldots, n\}$, there exists a constant of $a$ satisfying $a_{i k}-a_{j k}=a$.

Proof. (1) Suppose that $A$ is a fuzzy consistent judgment matrix such that

$$
a_{i j}=a_{i k}-a_{j k}+0.5,
$$

for any $i, j$ and all $k$. Therefore, we have

$$
a_{i k}-a_{j k}=a_{i j}-0.5=a .
$$

(2) Suppose that for any given $i, j$, there exists a constant of $a$ for each $k$ such that

$$
a_{i k}-a_{j k}=a,
$$

and when $j=k$, we have

$$
a_{i j}-a_{j j}=a
$$

Consequently, we get

$$
a_{i k}-a_{j k}=a_{i j}-a_{j j}
$$

Since $a_{i j}+a_{j i}=1$, so we can get $a_{j j}=0.5$. Finally, we obtain

$$
a_{i j}=a_{i k}-a_{j k}+0.5 \text {. }
$$

Property 2. Assume $A$ is a fuzzy complementary judgment matrix, we define a kind of fuzzy transform:

$$
\begin{gathered}
F: A \longrightarrow R, \\
a_{i j} \longrightarrow r_{i j}=\frac{r_{i}-r_{j}}{\alpha}+0.5, \quad \alpha \geq 2(n-1),
\end{gathered}
$$

where $r_{i}=\sum_{j=1}^{n} a_{i j}, i=1,2, \ldots, n$. Then, $R$ is a fuzzy consistent judgment matrix.

Proof. Firstly, we prove that $R$ is a fuzzy matrix. Since $0 \leq$ $a_{i j} \leq 1$, we know that

$$
\begin{gathered}
0.5 \leq \sum_{k=1}^{n} a_{i k} \leq n-0.5, \\
0.5-n \leq-\sum_{k=1}^{n} a_{j k} \leq-0.5 .
\end{gathered}
$$

Therefore, we have $\left|r_{i}-r_{j}\right| \leq n-1$. When $\alpha \geq 2(n-1)$, we get $0 \leq r_{i j} \leq 1$.

Secondly, we prove that $R$ is a fuzzy complementary matrix.

$$
r_{i j}+r_{i j}=\left(\frac{r_{i}-r_{j}}{\alpha}+0.5\right)+\left(\frac{r_{j}-r_{i}}{\alpha}+0.5\right)=1 .
$$
matrix

Finally, we prove that $R$ is a fuzzy consistent judgment

$$
\begin{aligned}
r_{i k}-r_{j k}+0.5 & =\left(\frac{r_{i}-r_{k}}{\alpha}+0.5\right)-\left(\frac{r_{j}-r_{k}}{\alpha}+0.5\right)+0.5 \\
& =\frac{r_{i}-r_{j}}{\alpha}+0.5=r_{i j} .
\end{aligned}
$$

Property 3. Assume that $A=\left(a_{i j}\right)_{n \times n}$ is a fuzzy complementary judgment matrix, and $w=\left(w_{1}, w_{2}, \ldots, w_{n}\right)$ is the weight vector or ordering vector, then 
TABLE 1 : The quantity scale of $0.1 \sim 0.9$.

\begin{tabular}{|c|c|c|}
\hline Scale value & Definition & Explanation \\
\hline 0.5 & Equally important & Two elements compared, equally important. \\
\hline 0.6 & Slightly important & $\begin{array}{l}\text { Two elements compared, an element is more slightly } \\
\text { important than another element. }\end{array}$ \\
\hline 0.7 & Obviously important & $\begin{array}{l}\text { Two elements compared, an element is more } \\
\text { obviously important than another element. }\end{array}$ \\
\hline 0.8 & Much more important & $\begin{array}{l}\text { Two elements compared, an element is much more } \\
\text { strongly important than another element. }\end{array}$ \\
\hline 0.9 & Extremely important & $\begin{array}{l}\text { Two elements compared, an element is more } \\
\text { extremely important than another element. }\end{array}$ \\
\hline $0.1,0.2,0.3,0.4$ & Converse comparison & $\begin{array}{l}\text { If the } u_{i} \text { is compared with } u_{j} \text {, get the judgment value } \\
a_{i j} \text {, then } u_{j} \text { is compared with } u_{i} \text {, the judgment value } \\
\text { is } a_{j i}=1-a_{i j} \text {. }\end{array}$ \\
\hline
\end{tabular}

TABLE 2: The statistical data of 12 RMUs in 2011.

\begin{tabular}{|c|c|c|c|c|c|c|c|c|c|c|c|c|}
\hline \multirow{2}{*}{ RMU } & \multicolumn{2}{|c|}{$u_{1}$} & \multicolumn{4}{|c|}{$u_{2}$} & \multicolumn{6}{|c|}{$u_{3}$} \\
\hline & $u_{11} \%$ & $u_{12} \%$ & $u_{21} \%$ & $u_{22} \%$ & $u_{23} \%$ & $u_{24} \%$ & $u_{31} \%$ & $u_{32} \%$ & $u_{33} \%$ & $u_{34} \%$ & $u_{35} \%$ & $u_{36} \%$ \\
\hline 1 & 99.8 & 96.3 & -25.8 & -30.3 & -64.2 & 89.3 & 92.4 & 98.5 & 91.5 & 96.1 & 108.4 & 77.6 \\
\hline 2 & 100.0 & 98.7 & 7.4 & 7.9 & 6.2 & 91.0 & 90.2 & 98.2 & 92.1 & 96.4 & 103.8 & 80.0 \\
\hline 3 & 99.7 & 96.5 & 10.5 & 15.9 & -1.3 & 92.7 & 93.0 & 98.4 & 93.2 & 95.8 & 97.7 & 82.5 \\
\hline 4 & 100.3 & 99.0 & 11.1 & 17.0 & 66.2 & 95.3 & 95.2 & 99.0 & 93.9 & 96.7 & 106.7 & 84.8 \\
\hline 5 & 99.9 & 96.7 & 3.5 & 20.2 & 28.8 & 92.2 & 89.8 & 98.1 & 92.6 & 93.4 & 98.6 & 81.4 \\
\hline 6 & 99.3 & 97.4 & 1.1 & 2.5 & 20.9 & 87.2 & 92.1 & 98.1 & 91.9 & 96.5 & 96.5 & 76.5 \\
\hline 7 & 99.5 & 98.2 & 6.0 & 8.5 & 76.0 & 90.7 & 90.5 & 98.4 & 89.4 & 97.0 & 97.4 & 79.3 \\
\hline 8 & 100.1 & 99.1 & -19.9 & -22.3 & -15.8 & 82.0 & 90.8 & 98.3 & 95.0 & 91.9 & 104.9 & 75.7 \\
\hline 9 & 100.0 & 97.9 & 20.4 & 21.3 & 37.6 & 92.5 & 74.1 & 99.0 & 90.5 & 95.6 & 96.4 & 74.2 \\
\hline 10 & 100.0 & 96.6 & 0.7 & 1.2 & 45.9 & 90.4 & 88.5 & 99.0 & 88.3 & 95.3 & 105.6 & 72.2 \\
\hline 11 & 99.8 & 97.2 & 12.5 & 13.6 & 18.7 & 89.1 & 98.3 & 98.7 & 89.4 & 96.3 & 102.1 & 68.8 \\
\hline 12 & 99.6 & 97.0 & 52.4 & 79.7 & 64.6 & 86.3 & 69.8 & 98.7 & 88.0 & 86.6 & 106.3 & 69.2 \\
\hline
\end{tabular}

(1) when $A$ is consistent, by using the normalizing rank aggregation method, the weight is given by

$$
w_{i}=\frac{2}{n^{2}} \sum_{j=1}^{n} a_{i j}, \quad i=1,2, \ldots, n,
$$

(2) when $A$ is not entirely consistent, first carry out the fuzzy consistent transformation by (11), and then through the normalizing rank aggregation method, the weight is given by

$$
w_{i}=\frac{1}{n}-\frac{1}{\alpha}+\frac{2}{n \alpha} \sum_{j=1}^{n} a_{i j}, \quad \alpha \geq 2(n-1) .
$$

Proof. (1) When $A$ is completely consistent,

$$
\begin{aligned}
w_{i} & =\frac{\sum_{j=1}^{n} a_{i j}}{\sum_{i=1}^{n} \sum_{j=1}^{n} a_{i j}}=\frac{\sum_{j=1}^{n} a_{i j}}{\sum_{1 \leq i<j \leq n}^{n}\left(a_{i j}+a_{j i}\right)+0.5 n} \\
& =\frac{\sum_{j=1}^{n} a_{i j}}{n^{2} / 2}=\frac{2}{n^{2}} \sum_{j=1}^{n} a_{i j} .
\end{aligned}
$$

(2) When $A$ is not entirely consistent,

$$
\begin{aligned}
w_{i} & =\frac{\sum_{j=1}^{n} r_{i j}}{\sum_{i=1}^{n} \sum_{j=1}^{n} r_{i j}}=\frac{\sum_{j=1}^{n} r_{i j}}{n^{2} / 2} \\
& =\frac{\sum_{j=1}^{n}\left(\left(\left(r_{i}-r_{j}\right) / \alpha\right)+0.5\right)}{n^{2} / 2}=\frac{1}{n}-\frac{1}{\alpha}+\frac{2}{n \alpha} \sum_{j=1}^{n} a_{i j} .
\end{aligned}
$$

From the above analysis, the steps for determining the weights can be summarized in the following.

Step 1. The expert gives out the fuzzy complementary judgment matrix $A$ by using the pairwise comparison method based on the quantity scale of $0.1 \sim 0.9$ [9] (see Table 1).

Step 2. Check whether $A$ is consistent or not. If consistent, calculate the weights by (15); if not, calculate the weight by (16).

\section{Production Performance Appraisal Rating for RMUs}

In this section, we introduce the method of TOPSIS [19] to decide the comprehensive ranking of RMUs and use the 
TABLE 3: The standardized decision data.

\begin{tabular}{lcccccccccccc}
\hline RMU & $u_{11}$ & $u_{12}$ & $u_{21}$ & $u_{22}$ & $u_{23}$ & $u_{24}$ & $u_{31}$ & $u_{32}$ & $u_{33}$ & $u_{34}$ & $u_{35}$ & $u_{36}$ \\
\hline 1 & 0.5000 & 0.0000 & 0.0000 & 0.0000 & 0.0000 & 0.5489 & 0.7930 & 0.4444 & 0.5000 & 0.9135 & 1.0000 & 0.5500 \\
2 & 0.7000 & 0.8571 & 0.4246 & 0.3473 & 0.5021 & 0.6767 & 0.7158 & 0.1111 & 0.5857 & 0.9423 & 0.6167 & 0.7000 \\
3 & 0.4000 & 0.0714 & 0.4642 & 0.4200 & 0.4486 & 0.8045 & 0.8140 & 0.3333 & 0.7429 & 0.8846 & 0.1083 & 0.8563 \\
4 & 1.0000 & 0.9643 & 0.4719 & 0.4300 & 0.9301 & 1.0000 & 0.8912 & 1.0000 & 0.8429 & 0.9712 & 0.8583 & 1.0000 \\
5 & 0.6000 & 0.1429 & 0.3747 & 0.4591 & 0.6633 & 0.7669 & 0.7018 & 0.0000 & 0.6571 & 0.6539 & 0.1833 & 0.7875 \\
6 & 0.0000 & 0.3929 & 0.3440 & 0.2982 & 0.6070 & 0.3910 & 0.7825 & 0.0000 & 0.5571 & 0.9519 & 0.0083 & 0.4813 \\
7 & 0.2000 & 0.6786 & 0.4067 & 0.3527 & 1.0000 & 0.6541 & 0.7263 & 0.3333 & 0.2000 & 1.0000 & 0.0833 & 0.6563 \\
8 & 0.8000 & 1.0000 & 0.0754 & 0.0727 & 0.3452 & 0.0000 & 0.7368 & 0.2222 & 1.0000 & 0.5096 & 0.7083 & 0.4313 \\
9 & 0.7000 & 0.5714 & 0.5908 & 0.4691 & 0.7261 & 0.7895 & 0.1509 & 1.0000 & 0.3571 & 0.8654 & 0.0000 & 0.3375 \\
10 & 0.7000 & 0.1071 & 0.3389 & 0.2864 & 0.7853 & 0.6316 & 0.6561 & 1.0000 & 0.0429 & 0.8365 & 0.7667 & 0.2125 \\
11 & 0.5000 & 0.3214 & 0.4898 & 0.3991 & 0.5913 & 0.5338 & 1.0000 & 0.6667 & 0.2000 & 0.9327 & 0.4750 & 0.0000 \\
12 & 0.3000 & 0.2500 & 1.0000 & 1.0000 & 0.9187 & 0.3233 & 0.0000 & 0.6667 & 0.0000 & 0.0000 & 0.8250 & 0.0250 \\
\hline
\end{tabular}

TABLE 4

(a)

\begin{tabular}{lccc}
\hline & $u_{1}$ & $u_{2}$ & $u_{3}$ \\
\hline$u_{1}$ & 0.5 & 0.7 & 0.7 \\
$u_{2}$ & 0.3 & 0.5 & 0.5 \\
$u_{3}$ & 0.3 & 0.5 & 0.5 \\
\hline
\end{tabular}

(b)

\begin{tabular}{lll}
\hline & $u_{11}$ & $u_{12}$ \\
\hline$u_{11}$ & 0.5 & 0.5 \\
$u_{12}$ & 0.5 & 0.5 \\
\hline
\end{tabular}

(c)

\begin{tabular}{lllll}
\hline & $u_{21}$ & $u_{22}$ & $u_{23}$ & $u_{24}$ \\
\hline$u_{21}$ & 0.5 & 0.5 & 0.5 & 0.6 \\
$u_{22}$ & 0.5 & 0.5 & 0.5 & 0.6 \\
$u_{23}$ & 0.5 & 0.5 & 0.5 & 0.6 \\
$u_{24}$ & 0.4 & 0.4 & 0.4 & 0.5 \\
\hline
\end{tabular}

(d)

\begin{tabular}{lllllll}
\hline & $u_{31}$ & $u_{32}$ & $u_{33}$ & $u_{34}$ & $u_{35}$ & $u_{36}$ \\
\hline$u_{31}$ & 0.5 & 0.5 & 0.6 & 0.7 & 0.9 & 0.5 \\
$u_{32}$ & 0.5 & 0.5 & 0.6 & 0.7 & 0.9 & 0.5 \\
$u_{33}$ & 0.4 & 0.4 & 0.5 & 0.6 & 0.8 & 0.4 \\
$u_{34}$ & 0.3 & 0.3 & 0.4 & 0.5 & 0.7 & 0.3 \\
$u_{35}$ & 0.1 & 0.1 & 0.2 & 0.3 & 0.5 & 0.1 \\
$u_{36}$ & 0.5 & 0.5 & 0.6 & 0.7 & 0.9 & 0.5 \\
\hline
\end{tabular}

Euclidean distance to describe the proximity between two RMUs. However, the proximity among the RMUs is different, for this reason, we adopt the fuzzy clustering to classify the RMUs. In order to obtain the optimal classification, the Fstatistics is mentioned in this paper.

4.1. Comprehensive Ranking. Assume that there are $m$ RMUs and $n$ evaluation indicators, the decision data matrix is denoted by $X=\left(x_{i j}\right)_{m \times n}$. According to the method of
TOPSIS, the comprehensive ranking procedure for RMUs consists of the following steps.

Step 1. Standardize the decision data matrix. The standardized decision data matrix is denoted by $Y=\left(y_{i j}\right)_{m \times n}$, and the transformation formula are given in the following;

(a) when the $j$ th indicator is the benefit type,

$$
y_{i j}=\frac{x_{i j}-\min _{i}\left\{x_{i j}\right\}}{\max _{i}\left\{x_{i j}\right\}-\min _{i}\left\{x_{i j}\right\}} .
$$

(b) when the jth indicator is the cost type,

$$
y_{i j}=\frac{\max _{i}\left\{x_{i j}\right\}-x_{i j}}{\max _{i}\left\{x_{i j}\right\}-\min _{i}\left\{x_{i j}\right\}} .
$$

(c) when the $j$ th is the target type,

$$
y_{i j}=1-\frac{\left|x_{i j}-x_{0}\right|}{\max \left\{\max _{i}\left\{x_{i j}\right\}-x_{0}, x_{0}-\min _{i}\left\{x_{i j}\right\}\right\}} \text {, }
$$

where $x_{0}$ is the target, and $\min _{i}\left\{x_{i j}\right\} \leq x_{0} \leq$ $\max _{i}\left\{x_{i j}\right\}$.

Step 2. Determine the weights of indicators. The weight vector $\omega=\left(\omega_{1}, \omega_{2}, \ldots, \omega_{n}\right)$ can be obtained by FAHP. Furthermore, we could calculate the weighted decision matrix $Z=$ $\left(z_{i j}\right)_{m \times n}$, where $z_{i j}=w_{j} \cdot y_{i j}$.

Step 3. Determine the positive ideal vector and the negative ideal vector. Respectively, denoted by $z^{+}=\left(z_{1}^{+}, z_{2}^{+}, \ldots, z_{n}^{+}\right) z^{-}=\left(z_{1}^{-}, z_{2}^{-}, \ldots, z_{n}^{-}\right)$, where $z_{j}^{+}=$ $\max _{i}\left\{z_{i j}\right\}, z_{j}^{-}=\min _{i}\left\{z_{i j}\right\}$.

Step 4. Calculate the Euclidean distance from the positive ideal vector and the negative ideal vector. The Euclidean distance between the $i$ th RMU and the positive ideal vector is denoted by

$$
d_{i}^{+}=\sqrt{\sum_{k=1}^{n}\left(z_{i k}-z_{k}^{+}\right)^{2}}
$$


TABLE 5: The weights of all the indicators.

\begin{tabular}{|c|c|c|c|c|}
\hline Indicator & Weights of indicators & Subindicators & Weights of subindicators & Combination weights \\
\hline \multirow{2}{*}{$u_{1}$} & \multirow{2}{*}{0.4222} & $u_{11}$ & 0.5 & 0.2111 \\
\hline & & $u_{12}$ & 0.5 & 0.2111 \\
\hline \multirow{4}{*}{$u_{2}$} & \multirow{4}{*}{0.2889} & $u_{21}$ & 0.2625 & 0.0758 \\
\hline & & $u_{22}$ & 0.2625 & 0.0758 \\
\hline & & $u_{23}$ & 0.2625 & 0.0758 \\
\hline & & $u_{24}$ & 0.2125 & 0.0614 \\
\hline \multirow{6}{*}{$u_{3}$} & \multirow{6}{*}{0.2889} & $u_{31}$ & 0.2056 & 0.0594 \\
\hline & & $u_{32}$ & 0.2056 & 0.0594 \\
\hline & & $u_{33}$ & 0.1722 & 0.0498 \\
\hline & & $u_{34}$ & 0.1388 & 0.0401 \\
\hline & & $u_{35}$ & 0.0722 & 0.0209 \\
\hline & & $u_{36}$ & 0.2056 & 0.0594 \\
\hline
\end{tabular}

TABLE 6: The relative closeness of 12 RMUs.

\begin{tabular}{lccc}
\hline RMU & $d_{i}^{+}$ & $d_{i}^{-}$ & $f_{i}^{*}$ \\
\hline 1 & 0.2762 & 0.1366 & 0.3309 \\
2 & 0.1224 & 0.2559 & 0.6765 \\
3 & 0.2495 & 0.1454 & 0.3683 \\
4 & 0.0606 & 0.3325 & 0.8459 \\
5 & 0.2223 & 0.1715 & 0.4355 \\
6 & 0.2723 & 0.1264 & 0.3170 \\
7 & 0.2054 & 0.1919 & 0.4829 \\
8 & 0.1477 & 0.2823 & 0.6565 \\
9 & 0.1448 & 0.2249 & 0.6083 \\
10 & 0.2252 & 0.1874 & 0.4542 \\
11 & 0.2065 & 0.1668 & 0.4468 \\
12 & 0.2450 & 0.1594 & 0.3941 \\
\hline
\end{tabular}

The Euclidean distance between the ith RMU and the negative ideal vector is denoted by

$$
d_{i}^{-}=\sqrt{\sum_{k=1}^{n}\left(z_{i k}-z_{k}^{-}\right)^{2}}
$$

Step 5. Calculate the relative closeness to the positive ideal vector. The relative closeness can be defined as

$$
f_{i}^{*}=\frac{d_{i}^{-}}{\left(d_{i}^{-}+d_{i}^{+}\right)}, \quad i=1,2, \ldots, m .
$$

Step 6. Decide the ranking according to the value of $f_{i}^{*}$. The bigger the closeness shows the better the ranking.

4.2. Rating of RMUs. Considering the gap between the differences in RMUs, the comprehensive ranking still is not enough. It is necessary to classify the RMUs with fuzzy clustering. Therefore, further we work out the distance matrix of RMUs, denoted by $D=\left(d_{i j}\right)_{m \times m}$, where

$$
d_{i j}=\frac{1}{n}\left|\sum_{k=1}^{n}\left(z_{i k}-z_{j k}\right)\right| .
$$

From (25), we can get the dynamic fuzzy clustering and the dynamic clustering figure.

In order to reasonably determine the number of classification, we introduce a kind of $F$ statistics [20]:

$$
F=\frac{\sum_{i=1}^{r} n_{i} \sum_{k=1}^{m}\left(\bar{x}_{i k}-\bar{x}_{k}\right)^{2} /(r-1)}{\sum_{i=1}^{r} \sum_{j=1}^{n_{i}} \sum_{k=1}^{m}\left(x_{i k}-\bar{x}_{j k}\right)^{2} /(n-r)} \sim F(r-1, n-r),
$$

where $r$ is the number of classification and $n_{i}$ is the number of elements in the $i$ th classification.

$$
\bar{x}_{i k}=\frac{1}{n_{i}} \sum_{j=1}^{n_{i}} x_{j k}, \quad k=1,2, \ldots, m
$$

is the average value of the $k$ th indicator in the ith classification.

$$
\bar{x}_{k}=\frac{1}{n} \sum_{j=1}^{n} x_{j k}, \quad k=1,2, \ldots, m
$$

is the average value of the $k$ th indicator of all the RMUs.

We can calculate the values of $F$ for each classification scheme by (26), under a given reliability $\alpha=0.05$, and find out the critical values of $F_{0.05}$. If $F>F_{0.05}$, the corresponding classification is feasible. Generally, take the classification number corresponding directly with $\max \{F-$ $\left.F_{0.05}\right\}$ as the optimal classification number and finally get the best classification rating.

\section{Example Analysis}

The statistical data of 12 reservoir management units (RMUs) of an oilfield in the year of 2011 are listed in Table 2.

According to the basic data in Table 2, we could obtain the evaluation results.

Step 1. Standardize the above decision data. The 12 indicators are all the benefit type; their standardized decision data are shown in Table 3. 
TABLE 7: The distance between any two RMUs.

\begin{tabular}{lcccccccccccccc}
\hline RMU & 1 & 2 & 3 & 4 & 5 & 6 & 7 & 8 & 9 & 10 & 11 \\
\hline 1 & 0.0000 & 0.0258 & 0.0097 & 0.0465 & 0.0117 & 0.0012 & 0.0154 & 0.0214 & 0.0221 & 0.0126 & 0.0122 & 0.0073 \\
2 & 0.0258 & 0.0000 & 0.0161 & 0.0207 & 0.0141 & 0.0245 & 0.0104 & 0.0044 & 0.0037 & 0.0132 & 0.0136 & 0.0185 \\
3 & 0.0097 & 0.0161 & 0.0000 & 0.0368 & 0.0021 & 0.0084 & 0.0058 & 0.0118 & 0.0125 & 0.0029 & 0.0026 & 0.0023 \\
4 & 0.0465 & 0.0207 & 0.0368 & 0.0000 & 0.0347 & 0.0452 & 0.0311 & 0.0250 & 0.0244 & 0.0339 & 0.0342 & 0.0392 \\
5 & 0.0117 & 0.0141 & 0.0021 & 0.0347 & 0.0000 & 0.0105 & 0.0037 & 0.0097 & 0.0104 & 0.0008 & 0.0005 & 0.0044 \\
6 & 0.0012 & 0.0245 & 0.0084 & 0.0452 & 0.0105 & 0.0000 & 0.0142 & 0.0202 & 0.0209 & 0.0113 & 0.0110 & 0.0061 \\
7 & 0.0154 & 0.0104 & 0.0058 & 0.0311 & 0.0037 & 0.0142 & 0.0000 & 0.0060 & 0.0067 & 0.0028 & 0.0032 & 0.0081 \\
8 & 0.0214 & 0.0044 & 0.0118 & 0.0250 & 0.0097 & 0.0202 & 0.0060 & 0.0000 & 0.0007 & 0.0089 & 0.0092 & 0.0141 \\
9 & 0.0221 & 0.0037 & 0.0125 & 0.0244 & 0.0104 & 0.0209 & 0.0067 & 0.0007 & 0.0000 & 0.0095 & 0.0099 & 0.0148 \\
10 & 0.0126 & 0.0132 & 0.0029 & 0.0339 & 0.0008 & 0.0113 & 0.0028 & 0.0089 & 0.0095 & 0.0000 & 0.0003 & 0.0053 \\
11 & 0.0122 & 0.0136 & 0.0026 & 0.0342 & 0.0005 & 0.0110 & 0.0032 & 0.0092 & 0.0099 & 0.0003 & 0.0000 & 0.0049 \\
12 & 0.0073 & 0.0185 & 0.0023 & 0.0392 & 0.0044 & 0.0061 & 0.0081 & 0.0141 & 0.0148 & 0.0053 & 0.0049 & 0.0000 \\
\hline
\end{tabular}

TABLE 8: The values of $F$ and the critical values of $F_{0.05}$.

\begin{tabular}{lcccccccccc}
\hline Classification Number & 2 & 3 & 4 & 5 & 6 & 7 & 8 & 9 & 10 & 11 \\
\hline$F$ & 0.77 & 1.79 & 1.63 & 15.06 & 28.50 & 1.81 & 1.42 & 2.32 & 0.24 & 0.79 \\
$F_{0.05}$ & 4.96 & 4.26 & 4.07 & 4.12 & 4.39 & 4.95 & 6.09 & 8.85 & 19.39 & 241.88 \\
$\Delta F=F-F_{0.05}$ & -4.19 & -2.47 & -2.44 & 10.94 & 24.12 & -3.14 & -4.67 & -6.52 & -19.14 & -241.09 \\
\hline
\end{tabular}

Step 2. Get the judgment matrixes of all the hierarchies through expert scoring Table 4.

Therefore, we can calculate the weights for the evaluation indicators shown in Table 5.

Step 3. Calculate the relative closeness of every RMU (see Table 6).

From Table 6, we know the comprehensive ranking as follows:

$$
\begin{aligned}
f_{4}^{*} & \succ f_{2}^{*} \succ f_{8}^{*} \succ f_{9}^{*} \succ f_{7}^{*} \succ f_{10}^{*} \succ f_{11}^{*} \succ f_{5}^{*} \\
& \succ f_{12}^{*} \succ f_{3}^{*} \succ f_{1}^{*} \succ f_{6}^{*} .
\end{aligned}
$$

Step 4. Determine the best classification rating. First of all, by (25), calculate the distance between any two RMUs (see Table 7).

Next, we can draw the dynamic fuzzy clustering figure (see Figure 2).

Lastly, calculate the values of $F$ for every kind of classification by $F$-statistics. The values of $F$ are listed in Table 8.

From Table 8 , it can be seen that the best classification number is "six", namely,

$$
\{4\} \succ\{2\} \succ\{8,9\} \succ\{7\} \succ\{3,5,10,11,12\} \succ\{1,6\} .
$$

\section{Conclusions}

Through analyzing the actual situation in the process of oilfield development, we first present some practically feasible evaluation indicators and their computing method in the second section. In order to reasonably decide the

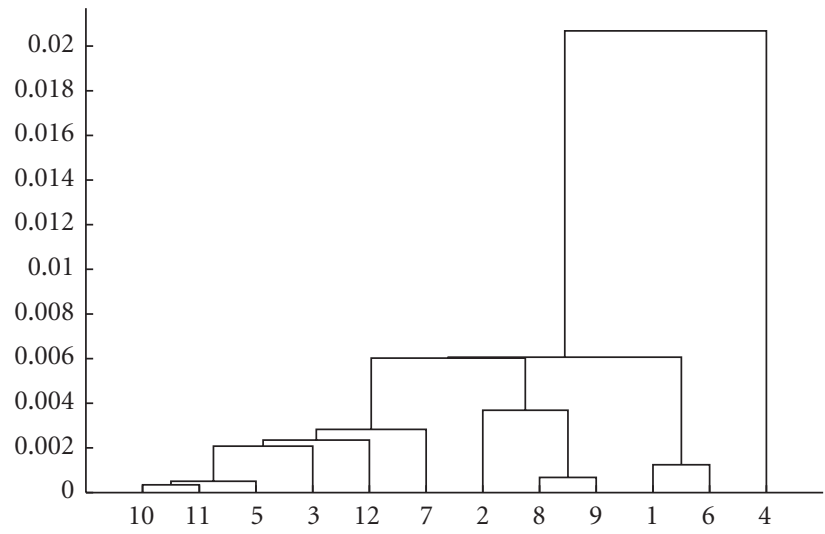

FIgure 2: The dynamic clustering figure.

weight of each indicator, we introduce a kind of fuzzy AHP in Section 3. Next, by means of the method of TOPSIS, it is easy to decide the comprehensive rankings of RMUs through calculating the weighted Euclidean distance between every RMU and the positive or negative ideal RMU. And we use the Euclidean distance to describe the proximity between two RMUs. Considering the proximity among the RMUs is different, the fuzzy clustering is introduced to classify the RMUs, and the production performance appraisal ratings of RMUs are determined by fuzzy clustering. In order to obtain the optimal classification, the $F$-statistics is mentioned in Section 4. Finally, a practical example is illustrated to explain the feasibility of this method.

In order to make the development department of oilfield companies accurately and timely grasp the current situation of oilfield development and management of RMUs, it needs to establish a relatively perfect evaluation method 
to really respond to the management level, efficiency, and development effect of RMUs, promoting the delicacy management of oilfield development. As we know, by means of the relatively effective evaluation method to ascertain the appraisal rating of the RMUs, we cannot only know their production performance, but also it is helpful to motivate the enthusiasm of practical production for all the RMUs. By using the evaluation method proposed in this paper, the management level could be constantly improved, and the delicacy management of oilfield development can be deepened. And the oilfield companies continuously strengthen the digital construction, so it will support the accuracy and objectivity of the evaluation method.

\section{References}

[1] Y. M. Chen, Reservoir Management, China University of Petroleum Press, DongYing, China, 2007.

[2] F. Jahn, M. Cook, and M. Graham, Hydrocarbon Exploration \& Production, vol. 55, Elsevier Science, Aberdeen, UK, 2nd edition, 2008.

[3] J. R. Fanchi, Integrated Reservoir Asset Management: Principles and Best Practices, Gulf Professional Publishing, Houston, Tex, USA, 2010.

[4] J. Y. Liu, Geological Foundation of Oil and Gas Field Development, Petroleum Industry Press, Beijing, China, 2006.

[5] P. T. Geoffrey, Oil and Gas: A Practical Handbook, Globe Law and Business, London, UK, 2009.

[6] N. Ezekwe, Petroleum Reservoir Engineering Practice, Prentice Hall, Upper Saddle River, NJ, USA, 2010.

[7] C. L. Li, Fundamentals of Reservoir Engineering, Petroleum Industry Press, Beijing, China, 2nd edition, 2011.

[8] Y. C. Li, The Technology of Petroleum Production, Petroleum Industry Press, Beijing, China, 2nd edition, 2009.

[9] J. J. Zhang, "Fuzzy analytic hierarchy process," A Chinese Journal of Fuzzy Systems and Mathematics, vol. 14, no. 2, pp. 80-88, 2000.

[10] Y. J. Lv, "Weight calculation method of fuzzy analytical hierarchy process," A Chinese Journal of Fuzzy Systems and Mathematics, vol. 16, no. 2, pp. 79-85, 2002.

[11] J. J. Zhang, "A new ranking method of fuzzy complementary judgment matrix," Operations Research and Management Science, vol. 14, no. 2, pp. 59-63, 2005.

[12] Y. J. Lu, W. L. Shi, and X. R. Guo, "The conditions of rank preservation and a general Priority formula for fuzzy complementary judgment matrix," Mathematics in Practice and Theory, vol. 39, no. 15, pp. 153-158, 2009.

[13] Z. P. Fan, J. Ma, and Q. Zhang, "An approach to multiple attribute decision making based on fuzzy preference information on alternatives," Fuzzy Sets and Systems, vol. 131, no. 1, pp. 101-106, 2002.

[14] F. Kong and H. Y. Liu, "Applying fuzzy analytic hierarchy process to evaluate success factors of E-commerce," International Journal of Information and Systems Sciences, vol. 1, pp. 406412, 2005.

[15] A. N. Haq and G. Kannan, "Fuzzy analytical hierarchy process for evaluating and selecting a vendor in a supply chain model," International Journal of Advanced Manufacturing Technology, vol. 29, no. 7-8, pp. 826-835, 2006.

[16] A. Azadeh, S. Nazari-Shirkouhi, L. Hatami-Shirkouhi, and A. Ansarinejad, "A unique fuzzy multi-criteria decision making: computer simulation approach for productive operators' assignment in cellular manufacturing systems with uncertainty and vagueness," International Journal of Advanced Manufacturing Technology, vol. 56, no. 1-4, pp. 329-343, 2011.

[17] W. G. Li, Q. Yu, and R. C. Luo, "Application of fuzzy analytic hierarchy process and neural network in power transformer risk assessment," Journal of Central South University, vol. 19, pp. 982-987, 2012.

[18] Z. B. Liu, W. Qing, and X. H. Kong, "Fuzzy comprehensive evaluation on the effect of measures operation for oil-water well," Advances in Fuzzy Systems, vol. 2011, Article ID 695690, 5 pages, 2011.

[19] D. Yong, "Plant location selection based on fuzzy TOPSIS," International Journal of Advanced Manufacturing Technology, vol. 28, no. 7-8, pp. 839-844, 2006.

[20] Y. D. Tan, M. Fornage, and H. Xu, "Ranking analysis of Fstatistics for microarray data," BMC Bioinformatics, vol. 9, article 142, 2008. 

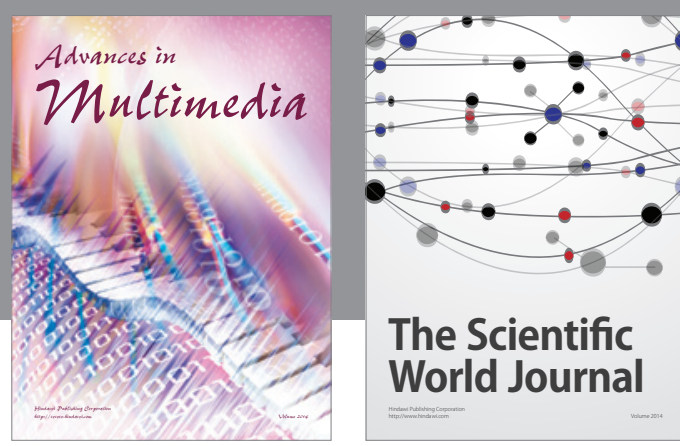

The Scientific World Journal
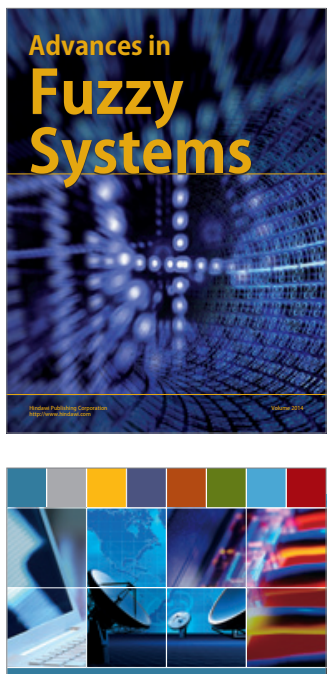

Computer Networks and Communications
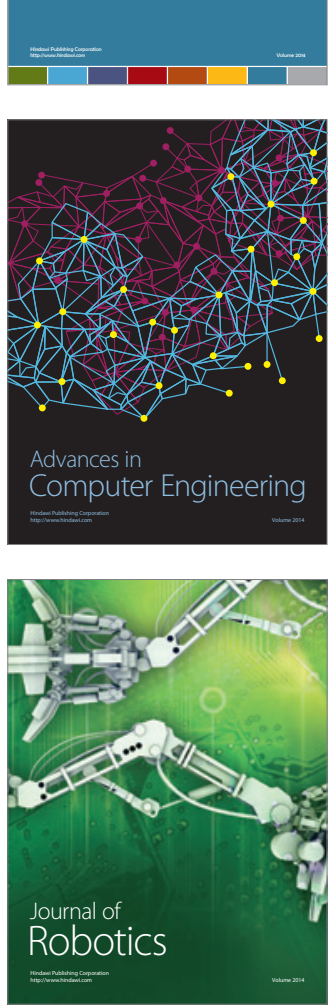
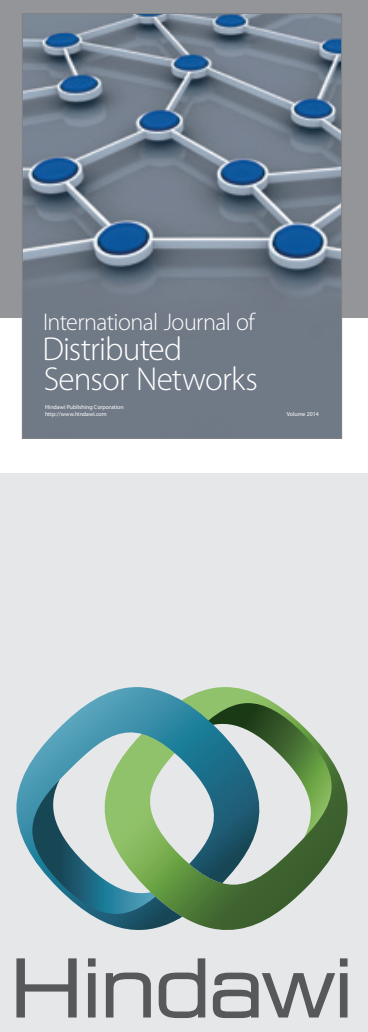

Submit your manuscripts at

http://www.hindawi.com
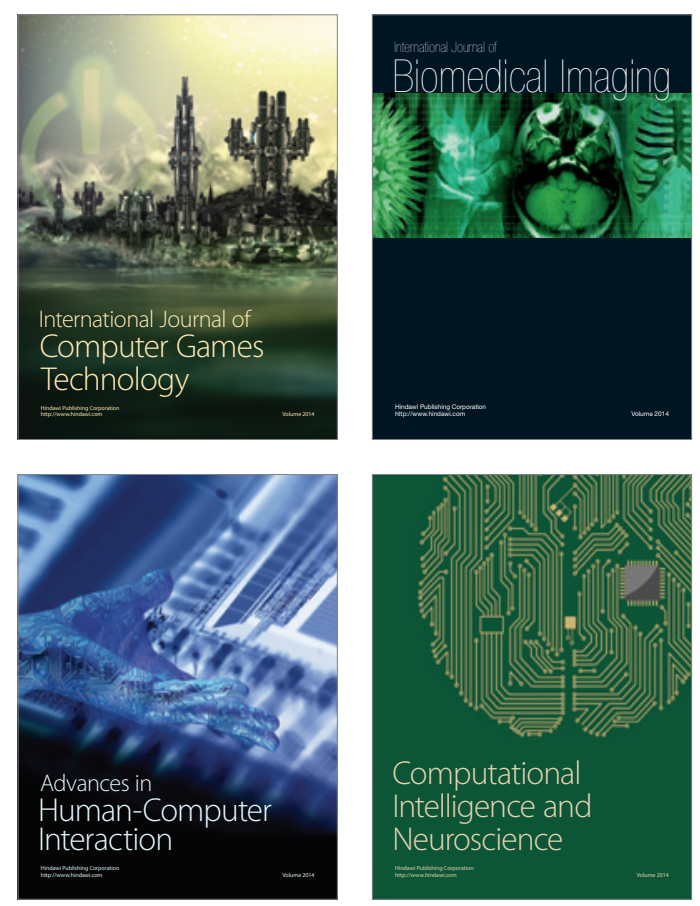
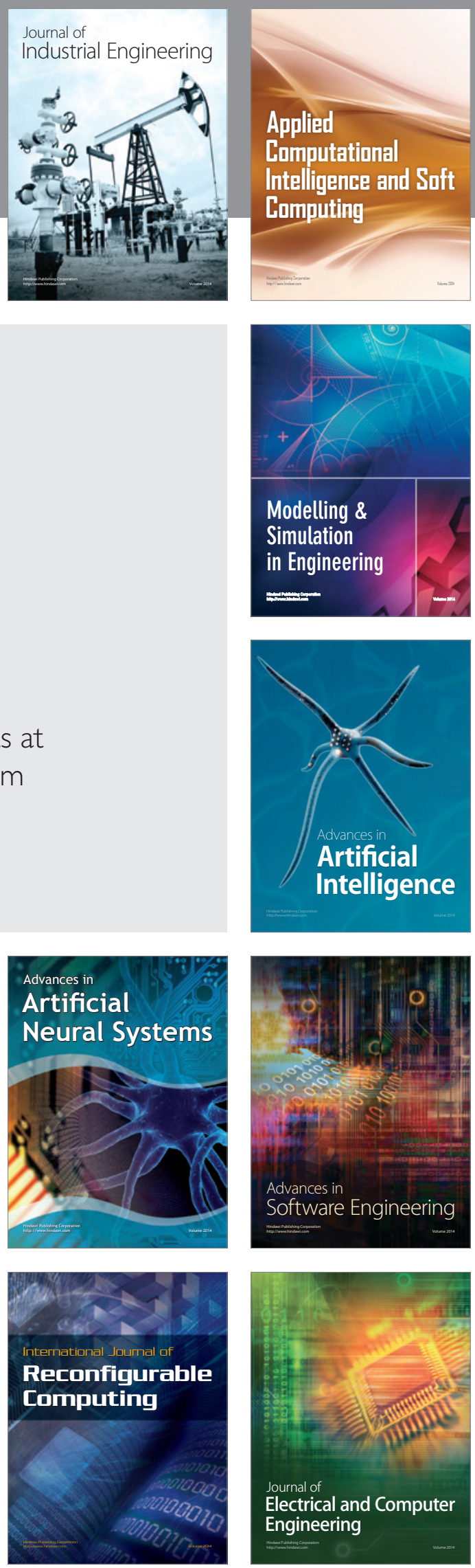